\title{
Reliability Evaluation of Voltage Source Converters for MVDC Applications
}

\author{
Gayan Abeynayake, Gen Li, Tibin Joseph, Wenlong Ming \\ Jun Liang \\ School of Engineering, Cardiff University, UK \\ \{AbeynayakePA, LiangJ1\}@ cardiff.ac.uk
}

\author{
Andrew Moon, Kevin Smith, James Yu \\ SP Energy Networks, Ochil House, Technology Park \\ High Blantyre, South Lanarkshire, Scotland, UK \\ amoon@spenergynetworks.co.uk
}

\begin{abstract}
The emerging Medium Voltage Direct Current (MVDC) distribution networks are becoming more attractive due to their flexible control of power flow and lower losses compared to traditional AC networks. This will significantly increase the wide uptake of renewable energy sources. Voltage source converters (VSCs) are the main building blocks of modern DC systems. Achieving a high reliability is an important aspect of selecting the converters for a specific project. However, it is still unclear which type of VSC is the most suitable solution for MVDC systems. This paper addresses this question from the reliability point of view. Three commonly used VSCs are considered for the analysis namely, two-level (2L), three-level NPC (3L-NPC), modular multi-level (MMC) converters and the cascaded three-level VSC which is currently being built for the first MVDC link in the UK. The results suggest that during the early stage of operation MMC shows a higher reliability. However, in the long run the other VSCs have shown higher reliabilities.
\end{abstract}

Index Terms-- Failure rate, mean time to failure, medium voltage DC, reliability, voltage source converters.

\section{INTRODUCTION}

For long distance power transmission, high-voltage directcurrent (HVDC) technology has proven to be the viable solution over AC transmission due to their lower capital costs, lower power losses and the capability to interconnect asynchronous grids [1]. Nevertheless, in medium-voltage (MV) distribution level, AC is still the predominant choice over DC [2]. However, with the rapid integration of decentralized renewable energy sources together with the growth of industrial and residential energy demand, AC distribution experience different sets of challenges. For instance, the congestion of distribution networks and unbalance conditions of three-phase AC network. These issues may result in system instability and force distribution network operators (DNOs) to expand and reinforce their grid infrastructures [3].

The MVDC technology has been considered as an attractive solution over MVAC due to their comparable benefits such as high-power transfer capacity, flexible power flow regulation and integrating DC sources and loads [4]. The optimum utilization of the existing assets is an important aspect in grid upgrading and planning. One feasible option is to convert existing MVAC lines into MVDC operation [5]. A practical

This work is supported in part by the European Union's Horizon 2020 research and innovation programme under the Marie Sklodowska-Curie grant agreement no. 765585, project title InnoDC; and in part by the Scottish Power Energy Network and OFGEM through the NIA project ANGLE-DC. example is the "ANGLE-DC" project which is the first MVDC link in the UK. The project aims to convert an existing $33 \mathrm{kV}$ MVAC line to $\pm 27 \mathrm{kV}$ MVDC link between Llanfair PG substation on Anglesey Island, and Bangor substation on the mainland in North Wales [6]. To this end, application of MVDC at different voltage levels have been proposed in the literature which ranges from $\pm 10 \mathrm{kV}$ to $\pm 70 \mathrm{kV}$ and with power transfer capacities between 30 to $150 \mathrm{MW}$ [7].

In HVDC level, use of modular multi-level converter (MMC) have advantages such as higher efficiency and modularity (which makes easy to scale to any voltage level) compared to two-level (2L) voltage source converter (VSC) and three-level neutral-point-clamped (3L-NPC) VSC. At low voltage DC level, it is still beneficial to use 2L VSC due to simpler design, control and low cost. However, at present shipboard power systems use thyristor-based rectifiers at MVDC level in the range of $20 \mathrm{kV}$ due to state-of-the-art and robustness [8]-[9]. Further, MVDC technology is identified as a feasible option for collection of offshore wind power and micro-grid applications in future.

In the light of this, evaluation of reliabilities of different VSCs is one of the important aspects when selecting a suitable topology at MVDC level. Because different VSC topologies have different IGBT arrangements which result in different reliability levels. Reliability of a converter can be improved by adding redundant IGBTs. However, there is a compromise between capital investment and cost incurred on energy-notserved due to converter failure. More redundant modules may also lead to more power losses and to higher system complexity. A VSC may fail to operate due to thermallyinduced stresses, cosmic ray effect or excessive fault currents of IGBTs [10] or due to failure of control and protection system, cooling system or power supply system. Thus, different methodologies have been used to assess failure modes of IGBTs as discussed in [11].

Reliability assessment of a converter is based on the stochastic behavior of IGBTs. Methods based on probability theories are necessary for evaluating a stochastic process. In [12], an analytical method was proposed to analyze the reliability of MMC at HVDC level with the consideration of different submodule (SM) arrangements and redundancy. However, a detailed evaluation of different converters suitable at MVDC levels has not been addressed in the open literature. 


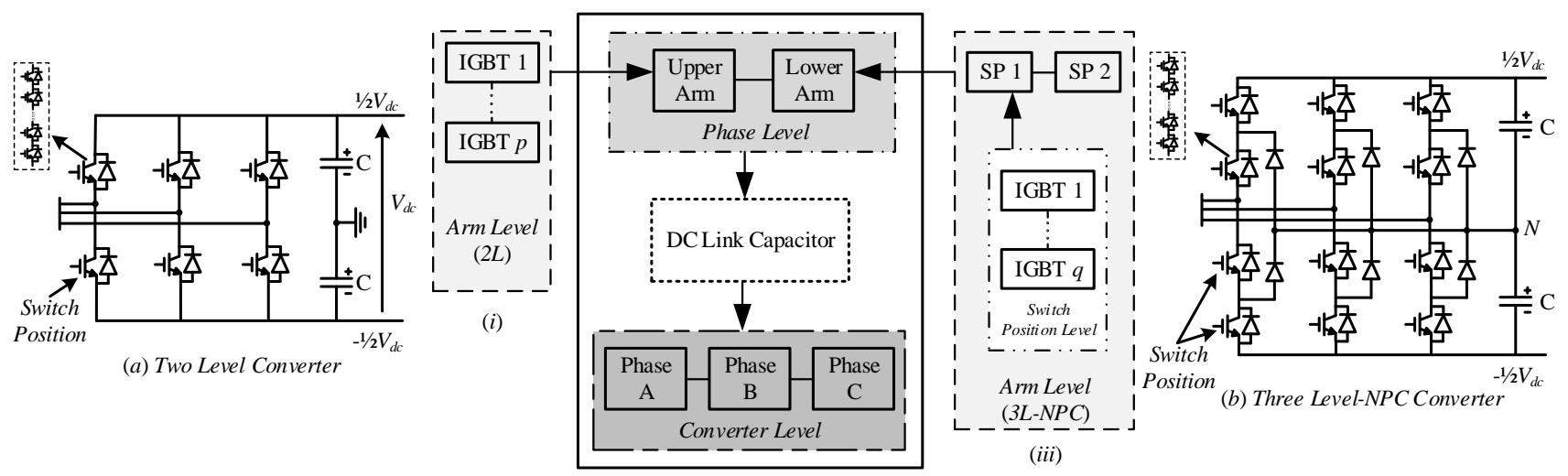

(ii)

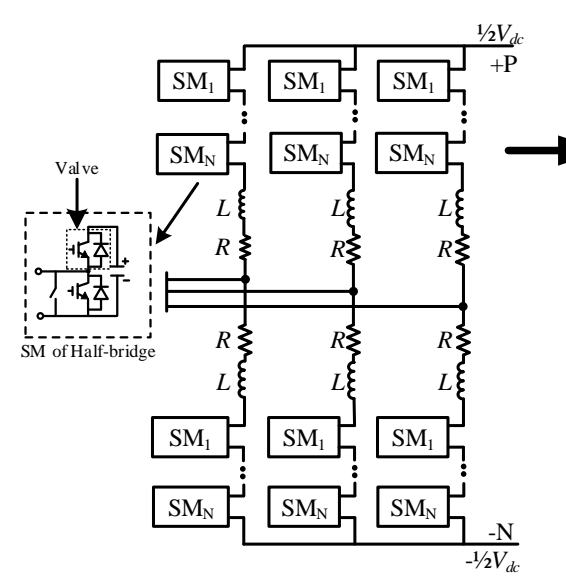

(c) Modular Multi-Level Converter

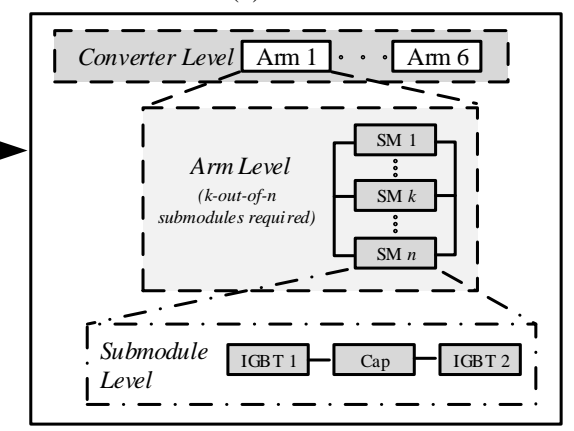

(iv)

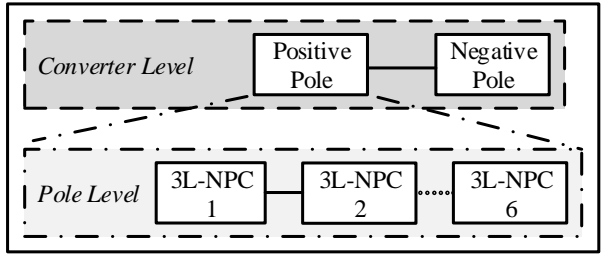

(v)

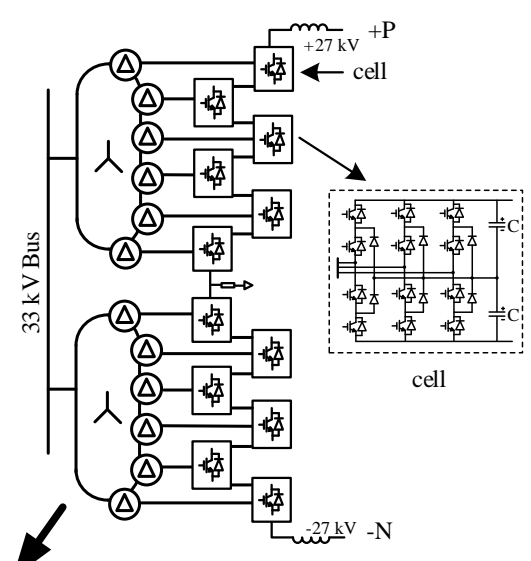

(d) ANGLE-DC Converter

Fig. 1. Different MVDC converter types and respective reliability block diagrams. (a) 2L-VSC; (b) 3L-NPC VSC; (c) MMC; (d) ANGLE-DC converter.

This paper provides a criterion for selecting a suitable VSC topology in terms of reliability. In this regard, reliability block diagrams (RBD) based reliability analysis is employed. Accurate modelling of RBD results in more accurate reliability analysis [13]. The studies in this paper are valuable for the selection of suitable converters in MVDC applications.

\section{RELIABILITY MODELLING OF CONVERTERS}

Reliability of a component is formally defined as the probability of performing its function satisfactorily for the period intended under the operating conditions encountered [13]. The reliability function of an IGBT module $R_{d}(t)$ (which normally represented by bathtub failure rate curve [14]) with a constant failure rate $\lambda_{d}$ is defined as

$$
R_{d}(t)=e^{-\lambda_{d} t} .
$$

In reliability analysis, the MTTF is used to evaluate the mean or expected time to failure $t$. Based on probability theory, the MTTF is represented by

$$
M T T F=\int_{0}^{\infty} R_{d}(t) d t .
$$

IGBT modules are typically classified into two types: wirebonded IGBT (WBI) modules and press-pack IGBT (PPI) modules. Upon a failure, WBIs will be open circuited (failopen mode) whereas PPIs will be short circuited (fail-short mode). Thus, PPIs are more reliable than classical WBIs in a series connection of IGBT modules [15]. However, WBI modules are still used in power electronics applications due to their lower cost. The WBIs are considered in this paper. Four types of VSC topologies, 2L, 3L-NPC, MMC and Angle DC converter (also called cascaded 3L-NPC) [6] were evaluated as shown in Fig 1.

In this paper, the series-connected IGBT group in the arms of $2 \mathrm{~L}$ and $3 \mathrm{~L}-\mathrm{NPC}$ is defined as switch position (SP), as shown in Figs. 1(a) and (b). The minimum number $k$, of required IGBT modules per SP can be calculated as follow

$$
k=\left\lfloor\frac{V_{d c}}{\alpha \times V_{I G B T}}\right\rfloor
$$

where $\alpha$ is the number of SPs per converter arm defined by

$$
\alpha=\beta-1
$$

where $\beta$ denotes the number of voltage levels of diodeclamped converter. In here, $\beta=2$ for $2 \mathrm{~L}$ and $\beta=3$ for $3 \mathrm{~L}-\mathrm{NPC}$ converter. The IGBT nominal voltage $V_{I G B T}$ is defined as

$$
V_{I G B T}=\eta \times V_{D}
$$


where $\eta$ is IGBT module de-rating factor and $V_{D}$ is IGBT withstand voltage.

\section{A. $2 L$ and $3 L-N P C$ Reliability Assessement}

Development of a correct RBD is necessary to evaluate reliability of a converter. Figs.1(a) and (b) illustrate the configurations of $2 \mathrm{~L}$ and $3 \mathrm{~L}-\mathrm{NPC}$ VSCs. It can be seen that there is only one SP in each arm of the 2L-VSC and two SPs in each arm of the 3L-NPC VSC. The number of IGBT modules in each SP can be calculated through (3). Then, the reliability $R_{s p}(t)$ of each SP is calculated as below

$$
R_{s p}(t)=\left[R_{d}(t)\right]^{k}=\left(e^{-\lambda_{d} t}\right)^{k} .
$$

The arm reliability $R_{a}(t)$ and phase reliability $R_{p}(t)$ are calculated as (7) and (8) respectively

$$
\begin{aligned}
R_{a}(t) & =\left[R_{s p}(t)\right]^{\alpha} \\
R_{p}(t) & =R_{a p}(t) \times R_{a n}(t)
\end{aligned}
$$

where $R_{a p}(t)$ and $R_{a n}(t)$ are the positive and negative arm reliabilities. The DC link capacitor reliability $R_{\text {cap }}(t)$ with failure rate of $\lambda_{\text {cap }}$ can be calculated using the same reliability evaluation method as of IGBT. The DC link capacitor $C_{d c}$ is estimated by method illustrated in [15].

$$
R_{\text {cap }}(t)=e^{-\lambda_{\text {cap }} t} \text {. }
$$

Finally, the converter reliability $R_{c}(t)$ for $2 \mathrm{~L}$ and $3 \mathrm{~L}-\mathrm{NPC}$ converters can be obtained by

$$
R_{c}(t)=\left[R_{p}(t)\right]^{3} \times R_{c a p}(t)
$$

Except the RBDs of the arms of 2L and 3L-NPC converters, the RDBs for phase level, DC link capacitor and converter are the same, as shown in Fig. 1(ii). The failure rate of the converter $\lambda_{c}(t)$ is given by

$$
\lambda_{c}(t)=-\frac{d\left[\ln R_{c}(t)\right]}{d t}
$$

\section{B. MMC Reliability Assessement}

There are two main MMCs: half-bridge (HB) and fullbridge (FB). As the capital costs and power losses of the FBMMC is much higher than the HB-MMC, the FB-MMC may not be an optimal option for MVDC applications. Therefore, only the HB-MMC is investigated. In terms of MMC redundancy design, there are two options namely active and passive modes. In the active mode, each arm comprises of $n$ SMs in which $n-k$ are the redundant SMs for converter's safe operation. Upon a failure of IGBT in a SM, it will be bypassed by the switch in the terminal of the SM (as shown in Fig.1(c)). Thus, each arm can be considered as a k-out-of-n system [12]. In the passive mode, the $n-k$ redundant SMs are bypassed during normal operation. When any IGBT fails in the operating $k$ SMs, a standby SM will be employed [17]. In this analysis, we consider the active mode as shown in Fig.1(iv).

To calculate number of SMs required for MMC, (3) can be used with $\alpha=1$ and $V_{I G B T}$ replaced with nominal voltage of the SM. Then, the reliability $R_{S M}(t)$ of a SM can be calculated as

$$
R_{S M}(t)=R_{I G B T, u}(t) \times R_{I G B T, l}(t) \times R_{c a p, S M}(t)
$$

where $R_{I G B T, u}(t)$ and $R_{I G B T, l}(t)$ are the reliabilities of upper and lower arms and $R_{c a p}, S M(t)$ is the reliability of the SM capacitance. The SM capacitance $C_{S M}$ is calculated by [18]

$$
C_{S M}=\frac{2 \times S_{M M C} \times E_{M M C}}{6 \times n \times V_{S M}^{2}}
$$

where $S_{M M C}$ is the MMC nominal capacity and $E_{M M C}$ the energyto-power ratio which is normally in the range of 30-40 kJ/MVA [19], $n$ the number of the SMs participate in switching and $V_{S M}$ the SM nominal voltage. The reliability $R_{a_{-} M M C}(t)$ of one arm can be calculated with probability theory applied for $k$-out-of- $n$ systems

$$
R_{a_{-} M M C}(t)=\sum_{i=k}^{n} C_{n}^{i}\left[R_{S M}(t)\right]^{i}\left[1-R_{S M}(t)\right]^{n-i}
$$

Finally, the MMC reliability $R_{M M C}(t)$ can be obtained by:

$$
R_{M M C}(t)=\left[R_{a_{-} M M C}(t)\right]^{6}
$$

\section{ANGLE-DC Converter Reliability Assessement}

The ANGLE-DC project uses a special kind of converter called cascaded 3L-NPC VSC to convert $33 \mathrm{kV} \mathrm{AC}$ to $\pm 27 \mathrm{kV}$ DC, as shown in Fig. 1(d). The converter comprises of 12 cells (pole-to-pole) and each cell is a 3L-NPC VSC with $4.5 \mathrm{kV}$ IGBT modules [20]. A high impedance DC grounding is applied in the neutral point of the converter [21]. Therefore, there is no single-pole operating mode of the converter. The cell reliability $R_{\text {cell }}(t)$ can be calculated using the same methodology described in Section II-A for 3L-NPC VSC and the converter reliability $R_{\text {Angle }}(t)$ can be calculated

$$
R_{\text {Angle }}(t)=\left[R_{\text {cell }}(t)\right]^{12} \text {. }
$$

\section{CASE STUdY}

A detailed analysis was performed to evaluate reliabilities of different converters with different MVDC levels and IGBT modules. To cover the full medium voltage range, three MVDC levels $\pm 10 \mathrm{kV}, \pm 27 \mathrm{kV}$ and $\pm 50 \mathrm{kV}$ were chosen. A common nominal converter capacity of $33 \mathrm{MVA}$ which is the rated capacity of ANGLE-DC converter [21] has been used to compare the results on a common ground. IGBT modules with blocking voltages of $4.5 \mathrm{kV}$ and $3.3 \mathrm{kV}$ and a derating factor of $56 \%$ [17] were selected. Hence, the IGBT nominal voltages are calculated using (5) with $2.52 \mathrm{kV}$ and $1.85 \mathrm{kV}$ for $4.5 \mathrm{kV}$ and $3.3 \mathrm{kV}$ IGBTs respectively. To calculate DC link capacitance for 2L, 3L-NPC and ANGLE-DC converters and SM capacitance of the MMC, energy-to-power ratio of 20 $\mathrm{kJ} / \mathrm{MVA}$ [10] and $30 \mathrm{~kJ} / \mathrm{MVA}$ [19] have been assumed.

The IGBT failure rates of $4.5 \mathrm{kV}$ and $3.3 \mathrm{kV}$ IGBTs were assumed as 0.0040 occ/yr [12] and 0.0035 occ/yr [22] respectively. Film capacitor, which is used in industrial applications due to their comparatively higher lifetime compared to electrolytic capacitor, was selected with failure rate of $0.001752 \mathrm{occ} / \mathrm{yr}$ [23]. For reliability analysis of MMCs, $5 \%$ redundant SMs per arm were chosen. All the reliability calculations were performed using MATLAB R2017a. 
However, change of converter reliabilities due to annual overhaul is not counted for calculations in this paper. Table I. shows the required number of IGBT modules per arm for different converters and voltage levels calculated using (3)-(5). Owing to rounding-up of value $k$ in (3), number of IGBTs per arm are slightly different for some $2 \mathrm{~L}$ and $3 \mathrm{~L}-\mathrm{NPC}$ converters.

TABLE I

REQUIRED NUMBER OF IGBTS PER ARM FOR DIFFERENT CONVERTERS

\begin{tabular}{l|c|c|c|c|c|c}
\hline \hline DC voltage (kV) & \multicolumn{2}{|c|}{ \pm 10} & \multicolumn{2}{c|}{ \pm 27} & \multicolumn{2}{c}{ \pm 50} \\
\hline IGBT (kV) & 4.5 & 3.3 & 4.5 & 3.3 & 4.5 & 3.3 \\
\hline 2-Lenverter & 8 & 11 & 22 & 30 & 40 & 55 \\
\hline 3-Level NPC & 8 & 12 & 22 & 30 & 40 & 56 \\
\hline MMC & 18 & 24 & 48 & 64 & 84 & 114 \\
\hline \hline
\end{tabular}

\section{A. Mean Time to Failure of Converters}

MTTF provides the average time that a converter will function before it fails. Fig. 2. illustrates the MTTFs (in years) for different MVDC converters. It can be seen from the graph that, with the increase of DC voltage level MTTFs decrease. This is due to utilization of higher number of IGBT modules. Also, the MTTF of the MMC for all the voltage levels are comparatively higher than the other converters due to the addition of redundant SMs. However, for $2 \mathrm{~L}$ and $3 \mathrm{~L}-\mathrm{NPC}$ converters with $3.3 \mathrm{kV}$ IGBT, the MTTF are slightly different due to utilisation of different number of IGBTs in each arm (except for $\pm 27 \mathrm{kV}$ scenario). For the MMC using $4.5 \mathrm{kV}$ IGBTs, their MTTF decreases starting from 7.6 to 3.0 years with the increase of MVDC level. Whereas for the MMC using $3.3 \mathrm{kV} \mathrm{IGBT}$, their MTTF decreases from 6.2 to 2.4 years. However, MTTF can be improved by utilising higher number of redundant SMs at the expense of capital cost.

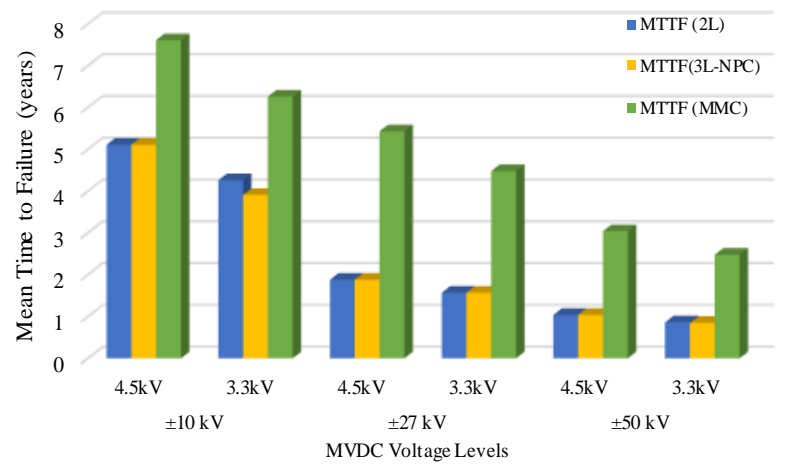

Fig. 2. MTTFs of different MVDC converters.

\section{B. Reliability Comparisons of Converters with $\pm 27 \mathrm{kV}$}

\section{1) Reliability of $2 L, 3 L-N P C$ and $M M C$}

Based on the reliability evaluation procedure described in Section II, failure rates of $\pm 27 \mathrm{kV}$ converters have been calculated and illustrated in Fig. 3. For both $2 \mathrm{~L}$ and $3 \mathrm{~L}-\mathrm{NPC}$ converters, a constant failure rate of $0.53 \mathrm{occ} / \mathrm{yr}$ and $0.63 \mathrm{occ} / \mathrm{yr}$ have shown for $4.5 \mathrm{kV}$ and $3.3 \mathrm{kV}$ IGBTs respectively. Since there are no redundant IGBT modules for these converters they are more susceptible to fail from the very beginning compared to MMC. However, for all $4.5 \mathrm{kV}$ IGBT based converters, the MMC shows better performance compared to $2 \mathrm{~L}$ and $3 \mathrm{~L}-\mathrm{NPC}$ converters before the first 8.8 years. For $3.3 \mathrm{kV}$ IGBT based
MMC, it has shown better reliability compared to their $2 \mathrm{~L}$ and 3L-NPC counterparts before 5.7 years. These results suggest that MMC reliability can be improved with utilisation of IGBTs with higher blocking capabilities.

The intersection points of different curves provide valuable information regarding how frequently the converter maintenance should be carried out over other. This can be taken as one of the parameters for the selection of the MVDC converter. It should be mentioned that the converter will be shut down for maintenance every 1 or 2 years. The failed components will be replaced in maintenance period.

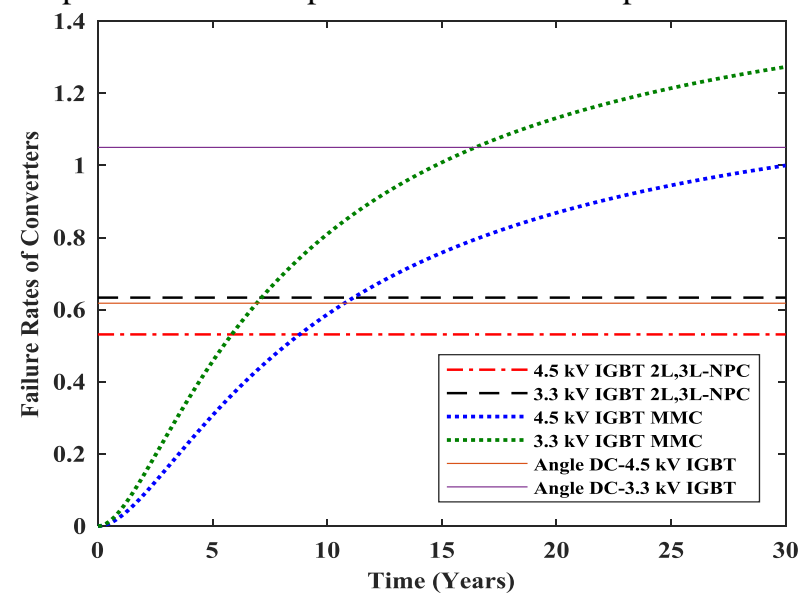

Fig. 3. Different failure rates for $\pm 27 \mathrm{kV}$ MVDC converter.

2) Reliabilty of ANGLE-DC converter

Additionally, the reliability of ANGLE-DC converter was performed with $4.5 \mathrm{kV}$ IGBT and with $3.3 \mathrm{kV}$ IGBT as a case study. The required number of IGBT modules and converter reliability were calculated as described in Section II-C. It can be noted that the failure rate of $3.3 \mathrm{kV}$ IGBT based ANGLE$\mathrm{DC}$ converter has the lowest reliability with the failure rate of $1.05 \mathrm{occ} / \mathrm{yr}$, while the $4.5 \mathrm{kV}$ IGBT based ANGLE-DC converter's failure rate is 0.56 occ/yr. The $2 \mathrm{~L}$ and $3 \mathrm{~L}-\mathrm{NPC}$ converters with the $4.5 \mathrm{kV}$ IGBTs shows lower failure rate than the ANGLE-DC converter.

However, the ANGLE-DC converter shows better AC harmonic performance at converter grid-side than its $2 \mathrm{~L}$ and 3L-NPC counterparts due to the cascaded configuration. Although the MMC has better reliability performance than the ANGLE-DC converter, MMC's higher capital cost and control system complexity could limit the potential application in MV level such as the one discussed.

\section{Reliability Comparison of $\pm 10 \mathrm{kV}$ and $\pm 50 \mathrm{kV}$ Converters}

To evaluate the change of reliabilities with different MVDC levels, the analysis was extended for $\pm 10 \mathrm{kV}$ and $\pm 50 \mathrm{kV}$ as shown in Figs. 4(a) and (b) respectively. The analysis was carried out following the same calculation procedure described in Section II. It can be noted that use of $3.3 \mathrm{kV}$ IGBT for $2 \mathrm{~L}$ and 3L-NPC converters have slightly different reliabilities due to the use of different number of IGBTs per arm. The use of 4.5 $\mathrm{kV}$ IGBT for 2L, 3L-NPC converters will result in higher converter reliability. However, converter failure rate will increase with the voltage level as shown in the two figures. The MMCs show better reliability performance than their counterparts in the initial 3 5 years. 


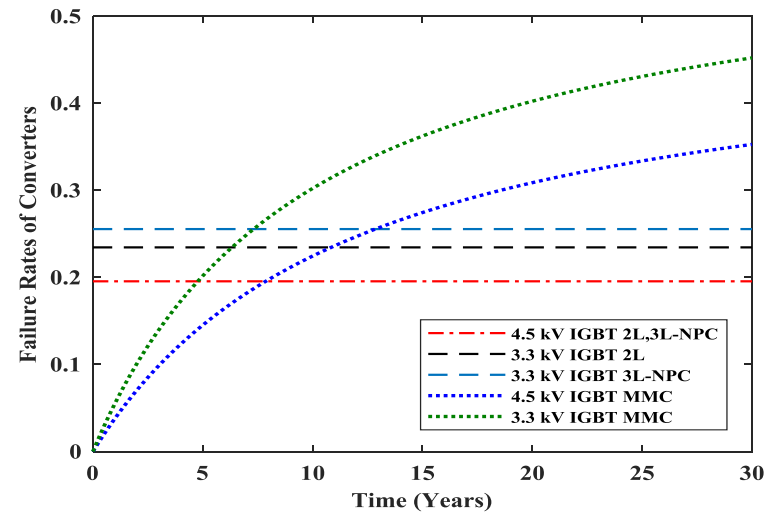

(a)

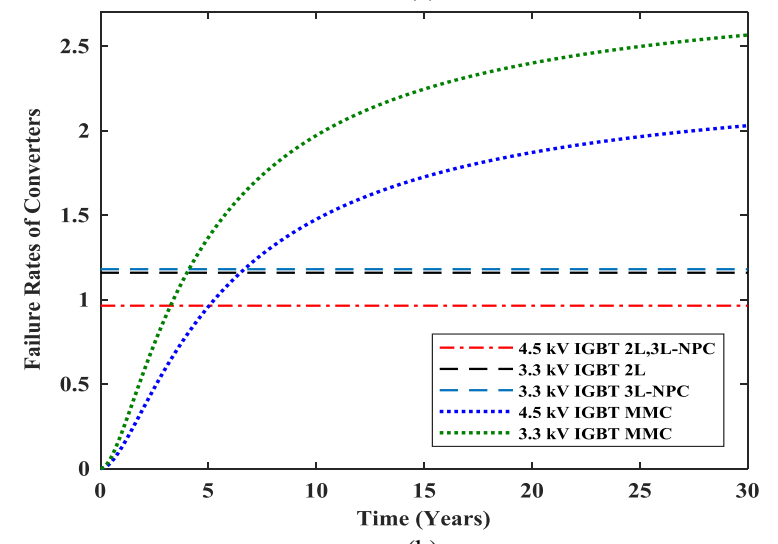

(b)

Fig. 4. Failure rate with different voltage levels. (a) $\pm 10 \mathrm{kV}$ converters; (b) \pm 50 $\mathrm{kV}$ converters.

\section{CONCLUSION}

A detailed reliability analysis of MVDC converters has been performed with the consideration of different converter topologies (2L-VSC, 3L-NPC VSC, MMC and ANGLE-DC converter) and three different MVDC levels. Due to the utilization of additional SMs, the MTTFs of the HB-MMC are higher than its counterparts. However, converter failure rates increase with the DC voltage level irrespective of the topologies. This could be minimized by utilizing IGBTs with higher blocking capabilities. MMC has shown better reliability at the early stage of operation but in the long run $2 \mathrm{~L}$ and $3 \mathrm{~L}-$ NPC VSCs have shown higher reliabilities. Further, the use of IGBTs with fail-open mode is not recommended due to lower reliability at early years compared to MMC. However, use of press-pack IGBT with fail-short mechanism will improve the reliability of $2 \mathrm{~L}$ and $3 \mathrm{~L}-\mathrm{NPC}$ VSCs make them suitable for MVDC applications.

\section{REFERENCES}

[1] G. Li, C. Li and D. van. Hertem, "HVDC technology overview," in HVDC Grid: For Offshore and Supergrid of the Future, Wiley-IEEE Press, April, 2016, ch. 3, pp.45-78.
[2] F. Blaabjerg, Zhe Chen and S. B. Kjaer, "Power electronics as efficient interface in dispersed power generation systems," IEEE Trans. Power Electron., vol. 19, no. 5, pp. 1184-1194, Sept. 2004.

[3] L. Zhang, J. Liang, W. Tang, G. Li, Y. Cai and W. Sheng, "Converting AC distribution lines to DC to increase transfer capacities and DG penetration," IEEE Trans. Smart Grid. Early Access, January, 2018.

[4] J. M. Bloemink and T. C. Green, "Benefits of Distribution-Level Power Electronics for Supporting Distributed Generation Growth," IEEE Trans. Power Del.,, vol. 28, no. 2, pp. 911-919, April 2013

[5] Lundquist. J., et al., "Guide to the conversion of existing AC lines to DC operation," Cigré Technical Brochure 583-Working Group B2 41, 2014.

[6] J. Yu, K. Smith, et el, "Initial designs for the ANGLE DC project; converting existing AC cable and overhead line into DC operation," in 13th IET Conference on AC and DC Power Transmission, 2017.

[7] D. M. Larruskain, I. Zamora, et al, "Conversion of AC distribution lines into DC lines to upgrade transmission capacity," Electric Power Systems Research, vol. 81, no. 7, pp. 1341-1348, Mar 2011.

[8] V. Staudt, et al., "Fault Scenarios in DC Ship Grids: The advantages and disadvantages of modular multilevel converters," in IEEE Electrification Magazine, vol. 3, no. 2, pp. 40-48, June 2015.

[9] C. Su, K. Lin and C. Chen, "Power Flow and Generator-Converter Schemes Studies in Ship MVDC Distribution Systems," IEEE Trans. Ind. Appl., vol. 52, no. 1, pp. 50-59, 2016.

[10] D. Jovcic and K. Ahmed, High-Voltage Direct Current Transmission: Converters Systems and DC Grids. Hoboken, NJ, USA: Wiley, 2015.

[11] V. Smet et al., "Ageing and Failure Modes of IGBT Modules in HighTemperature Power Cycling," IEEE Trans. Ind. Electron., vol. 58, no. 10, pp. 4931-4941, Oct. 2011.

[12] J. Guo, J. Liang, et al., "Reliability Analysis of MMCs Considering Submodule Designs with Individual or Series-Operated IGBTs," IEEE Trans. Power Del., vol. 32, no. 2, pp. 666-677, April 2017.

[13] Billinton, R., and Allan R, N. "Reliability evaluation of engineering systems" Springer, New York, NY, USA, 1992

[14] G.A. Klutke, P. C. Kiessler, and M. Wortman, "A critical look at the bathtub curve," IEEE Trans. Rel., vol. 52, no. 1, pp. 125-129, Mar. 2003.

[15] U. Choi, F. Blaabjerg and K. Lee, "Study and Handling Methods of Power IGBT Module Failures in Power Electronic Converter Systems," IEEE Trans. Power Electron., vol. 30, no. 5, pp. 2517-2533, May 2015.

[16] D. Jovcic K. Ahmed High-Voltage Direct Current Transmission: Converters Systems and DC Grids USA NJ Hoboken:Wiley 2015.

[17] P. Tu, S. Yang and P. Wang, "Reliability and Cost based Redundancy Design for Modular Multilevel Converter," IEEE Trans. Ind. Electron. Early Access, January 2018.

[18] J. Peralta, H. Saad, S. Dennetiere, J. Mahseredjian, and S. Nguefeu, "Detailed and averaged models for a 401-level mmc-hvdc system," IEEE Trans. Power Del., vol. 27, no. 3, pp. 1501-1508, Jul. 2012.

[19] B. Jacobson, et al., "VSC HVDC transmission with cascaded two-level converters," in CIGRE' Session, 2010, pp. B4-B110.

[20] R. Jakob, C. Keller, G. Mohlenkamp and B. Gollentz, "3-Level high power converter with press pack IGBT," in 2007 European Conference on Power Electronics and Applications, Aalborg, 2007, pp. 1-7.

[21] T. Joseph, J. Liang, G. Li, et al., "Dynamic control of MVDC link embedded in distribution network: - Case study on ANGLE-DC," in 2017 IEEE Conference on Energy Internet and Energy System Integration (EI2), Beijing, 2017, pp. 1-6.

[22] White Paper-TD02000001E, "The Reliability of Neutral Point Clamped vs. Cascaded H-Bridge Inverters, " EATON CORPORATION

[23] R. Grinberg, G. Riedel, A. Korn, P. Steimer and E. Bjornstad, "On reliability of medium voltage multilevel converters," in 2013 IEEE Energy Conversion Congress and Exposition, Denver, CO, 2013, pp. 4047-4052. 